\title{
Does Patient Race/Ethnicity Influence Physician Decision-Making for Diagnosis and Treatment of Childhood Disruptive Behavior Problems?
}

\author{
Ann F. Garland • Robin Taylor • Lauren Brookman-Frazee • \\ Mary Baker-Ericzen • Rachel Haine-Schlagel • Yi Hui Liu • Sarina Wong
}

Received: 27 May 2014 / Revised: 26 August 2014 / Accepted: 24 October 2014 / Published online: 17 December 2014

(C) W. Montague Cobb-NMA Health Institute 2014

\begin{abstract}
Race/ethnic disparities in utilization of children's mental health care have been well documented and are particularly concerning given the long-term risks of untreated mental health problems (Institute of Medicine, 2003; Kessler et al. Am J Psychiatry 152:10026-1032, 1995). Research investigating the higher rates of unmet need among race/ethnic minority youths has focused primarily on policy, fiscal, and individual child or family factors that can influence service access and use. Alternatively, this study examines provider behavior as a potential influence on race/ethnic disparities in mental health care. The goal of the study was to examine whether patient (family) race/ethnicity influences physician diagnostic and treatment decision-making for childhood disruptive behavior problems. The study utilized an internet-based video vignette with corresponding survey of 371 randomly selected physicians from across the USA representing specialties likely to treat these
\end{abstract}

A. F. Garland · R. Taylor · L. Brookman-Frazee •

M. Baker-Ericzen $\cdot$ R. Haine-Schlagel

Child and Adolescent Services Research Center (CASRC),

San Diego, CA, USA

R. Taylor

e-mail: robinmcfarlandtaylor@gmail.com

L. Brookman-Frazee

e-mail: 1brookman@ucsd.edu

M. Baker-Ericzen

e-mail: mjbaker@rchsd.org

R. Haine-Schlagel

e-mail: rhaine@mail.sdsu.edu

\section{A. F. Garland $(\bowtie) \cdot$ S. Wong}

Department of School, Family \& Mental Health Professions,

School of Leadership and Education Sciences, University of San

Diego, 5998 Alcalá Park, San Diego, CA 92110, USA

e-mail: agarland@sandiego.edu

S. Wong

e-mail: sarinaw@sandiego.edu patients (pediatricians, family physicians, general and child psychiatrists). Participants viewed a video vignette in which only race/ethnicity of the mother randomly varied (nonHispanic White, Hispanic, and African American) and then responded to questions about diagnosis and recommended treatments. Physicians assigned diagnoses such as oppositional defiant disorder (48\%) and attention deficit disorder (63\%) to the child, but there were no differences in diagnosis based on race/ethnicity. The majority of respondents recommended psychosocial treatment $(98 \%)$ and/or psychoactive medication treatment $(60 \%)$, but there were no significant differences based on race/ethnicity. Thus, in this study using mock patient stimuli and controlling for other factors, such as insurance coverage, we did not find major differences in physician diagnostic or treatment decision-making based on patient race/ethnicity.

\footnotetext{
L. Brookman-Frazee

Department of Psychiatry, University of California,

San Diego, CA, USA
}

R. Haine-Schlagel

Department of Psychology,

San Diego State University, San Diego, CA, USA

Y. H. Liu

Department of Pediatrics, University of California,

San Diego, CA, USA

e-mail: yiliu@ucsd.edu

M. Baker-Ericzen

Rady Children's Hospital and Health Center,

San Diego, CA, USA 
Keywords Physician decision-making $\cdot$ Patient race/ethnicity $\cdot$ Psychoactive medications $\cdot$ Childhood disruptive behavior problems

\section{Introduction}

Disruptive behavior problems ("DBPs," e.g., aggression, impulsivity, oppositional and defiant behavior, etc.) are the most common presenting problems for children entering mental health care [3]. These problems are associated with several different diagnoses, and children with these problems are at elevated risk for a variety of maladaptive outcomes including serious mental illness and incarceration in adulthood [4]. There are more evidence-based treatments (psychosocial and psychopharmacological) for these problems than for other childhood mental health conditions [5]. Unfortunately, racial and ethnic minority youths are less likely than non-Hispanic White youths to receive these treatments given evidence of significant disparities in service access, use, and intensity [6-9]. These disparities in mental health care have not been explained entirely by potentially confounding factors such as family income, insurance coverage, or clinical need [7, 10], suggesting that there are many additional influences that need to be identified and addressed in order to ultimately reduce these disparities.

\section{Racial/Ethnic Disparities in Children's Mental Health Care}

While there is unmet need for mental health care for all children, numerous studies indicate that unmet need is significantly greater for racial and ethnic minority youth compared to non-Hispanic White youth [6-8]. Evidence suggests that African American, Latino, and Asian American youth are less likely to enter mental health care and even after entering care, youth from racial/ethnic minority groups obtain fewer and/or less intensive services [7, 11, 12]. Reducing these disparities in mental health care is a national priority and efforts to reduce disparities require accurate and comprehensive data identifying disparity determinants. Raphael and Beal have proposed a useful conceptual framework to guide disparity research that identifies potential determinants of disparities at multiple levels ranging from the individual level to the health systems (including providers), community, and societal levels [13]. In children's mental health care, much of the research has addressed individual factors, with important emerging research identifying culturally driven beliefs about mental illness and help-seeking preferences that may partially explain service disparities [14, 15]. Additional research has identified sociodemographic factors that are associated with service use (e.g., insurance coverage, geography, parental education level) $[10,16]$. However, very little research has been conducted on how factors at the health systems level, especially provider attitudes or behaviors, influence service use for diverse patients. Given that individual/family factors do not entirely explain disparities in mental health services [7], research on potential determinants at other levels in the Raphael and Beal framework is needed [13]. To complement existing research at the individual and family level, this study examines potential determinants of disparities at the health system level by examining how provider decision-making on diagnosis and treatment recommendations may vary based on patient race/ethnicity.

While providers' decision-making has been scarcely examined, the limited research available suggests there may be bias in diagnostic decision-making by patient race/ethnicity in the mental health field. Studies of provider assigned psychiatric diagnoses reveal prevalence differences by race/ethnicity, with children of color (particularly African American youth) more likely to be diagnosed with psychotic disorders and nonHispanic Whites more likely to be diagnosed with mood disorders [17-19]. Given that there is no convincing evidence to support a valid racial/ethnic difference in prevalence of these disorders [11], bias in clinical decision-making is suspected. In a large national study, investigators found that African American youth were less likely to be identified with internalizing psychiatric disorders (including mood disorders) compared to non-Hispanic White youth [11]. Research on adult mental health also provides some evidence for potential bias. For example, Strakowski and colleagues found that mental health providers are more likely to attend to symptoms of psychosis for African American men compared to nonHispanic White men when all other factors are controlled [20]. Thus, the limited research on potential influence of patient race/ethnicity on physician diagnostic decisionmaking suggests that there may be an effect, but this has not been well tested for childhood behavior problems.

Racial disparities in physicians' psychoactive prescription patterns have also been identified. In one study of adult Medicaid beneficiaries, physicians prescribed newer atypical antipsychotic medications to non-Hispanic Whites more frequently compared to African American patients for whom they were more likely to prescribe traditional antipsychotics [21]. Race/ethnic differences in psychoactive medication use among children have been relatively well documented. Specifically, numerous studies have reported higher rates of psychoactive medication use among non-Hispanic White children and adolescents compared to racial/ethnic minority youths $[9,16,22]$. In our previous study of communitybased mental health care for children with DBPs, we found that two thirds of children ages 4-13 used psychoactive medications, but racial/ethnic minority youths were significantly less likely than non-Hispanic White youths to utilize these medications, even when controlling for potentially confounding factors [16]. In fact, family ethnicity was a more robust predictor of medication use than were clinical factors such as 
the child's diagnosis or behavior problem symptom severity. However, the extent to which these disparities in service use are driven by patient access, patient preferences, and/or provider treatment recommendation decisions is unknown.

Most of the research examining determinants of racial/ ethnic differences in psychoactive medication use among children has examined factors such as insurance coverage or differences in patient and family treatment preferences. For example, Yeh and colleagues have found that ethnic minority parents of children with mental health problems are less likely to endorse biopsychosocial (i.e., traditional Western medical) beliefs about the causes of emotional and behavioral problems, and correspondingly, they report less positive attitudes than non-Hispanic Whites about the potential benefits of biopsychosocial interventions, such as psychoactive medications [23]. Research in the adult physical health care domain suggests there may be complex interactions between patients' beliefs and preferences, providers' perceptions of patients' preferences (including potential biases), and provider clinical decision-making. Specifically, van Ryn and Fu hypothesize that provider beliefs about patients influence their interpretation of symptoms, which subsequently influence clinical decision-making [24]. This hypothesis is supported by their earlier research indicating that physicians' perceptions of patients' resources and/or attitudes predicted their treatment recommendations. The extent to which physicians may be making assumptions about patients' resources and attitudes based solely on their race is unknown. However, physicians were more likely to rate African American patients as lacking in social support and as more unlikely to participate in treatment compared to non-Hispanic White patients [25]. Yet independent of race, physicians' perceptions of patients' education and physical activity preferences were significant predictors of treatment recommendations [26]. Tamayo-Sarver and colleagues found that "socially desirable patient characteristics" associated with occupation, relationship with primary care doctor, and reliability of follow-up were significantly associated with opioid prescription rates, but race/ethnicity was not [27].

These mixed findings support the need for further investigation of the role of provider clinical decision-making as a determinant of health care disparities, and specifically, the extent to which patient race/ethnicity may drive clinical decision-making. Given the complex interactions between provider and patient that influence provider clinical decision-making, there is a great need for studies of provider behavior that can control for patient characteristics to test specific effects. Analog or mock patient presentations (e.g., text descriptions of clinical vignettes, "mock" patients presenting "in vivo," or video simulations) are used frequently to assess clinical decision-making for education, evaluation, and research purposes because these methods allow for control over patient variables. While there are potential limitations to this method and the extent to which one can be certain that provider decision-making based on mock stimuli reflects decisionmaking in actual practice, this method has significant advantages for research as it allows for standardization and experimental manipulation to test hypotheses. Several health care studies have effectively used vignettes as stimuli to elicit clinical decision-making outcomes. For example, Epstein and colleagues used videotape vignettes to examine physician characteristics associated with clinical decisions regarding treatment for depression [28]. They found that several physician characteristics (e.g., younger physician age) were significantly associated with quality of care indicators. Burk and Sher also used videotapes of adolescents to examine the impact of family history of alcoholism on mental health workers' assessment of diagnosis and predicted psychopathology [29]. In a study most closely reflecting the general aims of this study, Tamayo-Sarver and colleagues used text vignettes (varying only the name of the patient) with color face photos depicting patients of different races, to test for differences in emergency room physicians' prescription patterns as noted above [27].

\section{Children with Disruptive Behavior Problems}

The clinical problem area selected for this study is disruptive behavior in childhood because the vast majority of children entering public mental health care present with these types of problems [3]. Children who present with significant disruptive behavior problems may meet criteria for a variety of psychiatric diagnoses and there are multiple evidence-based psychosocial and psychopharmacological interventions for these patients. Behavioral parent training approaches, parent-child interaction therapies, and problem-solving skill training for children are a few of the most well-established evidence-based practices for these problems [30]. A variety of psychoactive medications are also used frequently for these patients. In fact, a national study indicates that the most common problem category for children receiving psychoactive medications in the USA is DBPs (including ADHD) [31] and national "best practice" recommendations include prescription of psychoactive medications for these youths [32]. Efficacy research data offer promising support for the use of psychoactive medications for children with DBPs, particularly stimulant medication for children with attention and hyperactivity symptoms, which often occur concurrently with other DBPs [33]. The majority of children treated with stimulants for ADHD show a positive response [34]. However, several studies indicate that non-Hispanic White children are much more likely to use these medications than are children from racial/ethnic minority backgrounds [16, 35]. The role of physician prescription patterns in driving these differences is unknown and is a major focus of this study. 
There is significant national attention to the increased use of psychoactive medications for children $[10,36]$ with public controversy about rising rates of use of psychoactive medications for children, including increasing rates of polypharmacy [31] and potential health risks. Several medications are recommended as "best practices," but there is considerable debate about potential over- and/or under-use and concern about unintended effects of several different psychoactive medications used to treat DBPs in childhood. There are specific concerns also about the dramatically rising rates of the use of atypical antipsychotics for children with a variety of behavioral problems and the physical health risks associated with these medications (including weight and metabolic effects) [31]. Within this debate, there has been limited attention to the role of racial/ethnic disparities in medication use, but such attention is highly relevant as it addresses potential factors other than clinical need that may be influencing utilization. Thus, studies of factors (both clinical and non-clinical) driving medication utilization are timely. The clinical problem area of DBPs provides an excellent focus for research on providers' clinical decision-making on medication because (a) it is so common that all providers should have some knowledge and opinions, as well as experience; and (b) while there is some consensus on diagnosis and recommended treatments, there is enough ambiguity regarding a range of potentially diverse treatment options to yield sufficient variability for study.

The primary aim of this study is to test for differences in physician diagnostic and treatment decision-making associated with patient family race/ethnicity (specifically African American, Hispanic, and non-Hispanic White (NHW)) holding other factors constant (such as clinical presentation, insurance coverage, treatment motivation, family history and context, etc.). Given the documented disparities in psychoactive medication use by patient race/ethnicity, the primary hypothesis is that physicians will be more likely to recommend prescription of psychoactive medications for NHW patients compared to African American and Hispanic patients even when other client characteristics are held constant.

\section{Research Methods}

Methods for this study were developed and piloted by a collaborative group of investigators and practicing physicians supported by a collaborative planning grant from the UCSD Division of Child Development and Community Health within the Department of Pediatrics. Methods are described below in two phases: phase 1-development and pilot testing of clinical vignettes and survey measure; phase 2 -implementation of national web-based physician survey. The study was approved by the university's human subjects committee.
Phase 1: Clinical Vignette Development

The goal was to produce three clinical video vignettes that differed only by race/ethnicity of the actor and represented (a) common clinical presentation in community care, and (b) sufficient ambiguity in diagnosis and treatment recommendations to elicit variability in physicians' decisions on the key outcome variables (diagnosis and treatment recommendations). A script for the vignette actors was developed collaboratively based on provider input (vignette script is included as an addendum). The video vignette portrayed a mother reporting on her child's symptoms and functioning, as well as some contextual information as described below. Specifically, the child's reported symptoms met DSM-IV criteria for oppositional defiant disorder (ODD; a common DBP diagnosis for children in public mental health services) plus some ADHD symptoms that were sub-threshold for diagnosis, to reflect the overlapping symptoms typically occurring in DBP populations.

The vignette presentation included controlled (i.e., consistent across all three vignettes) descriptive contextual data about the child, including age, gender, grade and performance in school, as well as data on family composition, school, and social functioning. The vignette also stated specifically that the family had optimal health insurance to cover treatment and that they were open to all treatment recommendations, controlling for treatment access and family motivation. The vignettes varied only by race/ethnicity of the parent (African American, Hispanic, and non-Hispanic White because these are the three groups that providers are most likely to have had some experience with across varying national locations).

Dozens of potential actors were screened (via photo submission and follow-up phone calls to most likely candidates). A total of eight actors were interviewed and screen tested to control for other potentially confounding variables such as age, articulateness, general attractiveness, and eye contact. Three actors representing each race/ethnic group were selected for final taping and video production. Each of the two subsequent actors watched the first video that was filmed and were instructed to match the pacing and emotional expressiveness. External factors such as clothing, jewelry, and scenery were standardized across vignettes. Several "takes" were conducted until a vignette was judged to be equivalent to the others. Videotaping and production was conducted by a professional Visual Media Group and all video was shot in high-definition.

The three final vignettes were distributed to 12 independent physicians for pilot-testing to rate each vignette actor on "articulateness," "believability," and "attractiveness" and to confirm intended race/ethnic representation. Viewers confirmed the intended race/ethnicity of the actor and used a 14 Likert scale to rate the constructs listed above. These ratings were used to assess the extent to which all three presentations 
were equivalent on factors that may influence physician decision-making. Mean ratings across all three race/ethnicity vignettes were within .25 points on all three constructs and thus determined equivalent. Equivalence across videos was considered essential to the study design to test for a specific isolated effect of patient race/ethnicity on physician decision-making.

\section{Phase 1: Survey Development}

Survey questions were developed by the collaborative group and refined based on piloting with practicing physicians. Three focus groups were held with local physicians to generate feedback on diagnostic and treatment planning challenges and factors that impact diagnostic and treatment planning decisions. Members of the focus groups were also asked to complete the survey and provide feedback on comprehension and feasibility.

The content of the 20-item final version of the survey (available from the first author upon request) can be characterized by five sections: demographic information, patient characteristics, clinical vignette presentation, diagnostic assignment, and treatment recommendations. Demographic questions assessed age, gender, race/ethnicity, medical school location, years in practice, medical specialty, practice setting, and location. Patient characteristic items assessed proportion of the physician's practice population that were pediatric, racial/ethnic minorities, unfunded/publicly funded, and had mental health or behavioral presenting problems. Diagnosis items asked respondents to select any DSM-IV diagnoses that should be assigned to the child described in the vignette. Possible responses included ADHD, anxiety disorder, autism spectrum disorder, bipolar disorder, conduct disorder, depression, disruptive disorder not otherwise specified, intermittent explosive disorder, oppositional defiant disorder, substance abuse, and none. The specific diagnostic categories selected were based on prevalence of diagnoses assigned to children with disruptive behavior problems. Treatment questions asked physicians if they would recommend psychosocial/ psychotherapeutic treatment (yes, no), and/or psychopharmacological treatment (yes/no), and if yes for either category, asked for selection of specific type of treatment from a range of options. Respondents also had the option to select "no" treatment or alternative holistic or complementary medicine treatments.

\section{Phase 1: Web-Based Survey Procedures}

A variety of web-based survey software options were reviewed and SurveyGizmo was selected. This platform was selected due to the innovative survey technologies, including the ability to embed streaming media, support random assignment of video vignette conditions using sophisticated branch logic techniques, and conversion of inputted data to a readable SPSS export for analysis. Viddler Video Platform was used for encoding, storing, and delivering the video vignette through SurveyGizmo. It was selected for its ability to host streaming video in MAC or PC formats, with full mobile iOS and Android device support to ensure reliable delivery to a wide variety of respondent conditions. Additionally, Viddler is a secure video host and prevents respondents from copying or tagging the video for sharing on social media sites.

Survey items were built into the web-based software and tested multiple times using a random response generator. This function creates hundreds of responses to the survey and aids in the identification of faulty skip logic, problems with text fields, and errors with encoding of streaming video. In addition to the random response testing, a small sample of physicians completed the web-based survey prior to launch of the national survey to test for functionality of email invitation, email embedded survey link, and streaming video vignette. These participants were also asked to note total time to completion of the survey, clarity of items, instructions, and skip logic patterns.

\section{Phase 2: National Web-Based Survey Administration}

\section{Participants}

Participant sample pools were randomly selected from the nationwide American Medical Association (AMA) member database maintained by Medical Marketing Services (MMS). MMS was contracted to deliver email survey invitations with an embedded survey link to AMA member physicians with active email addresses. Two waves of 1,000 physicians from each of the targeted specialties (Pediatrics, Psychiatry, Child and Adolescent Psychiatry, and Family Medicine) were randomly selected to receive the email invitation to participate in the survey. Because the number of members in each specialty group varies, MMS utilized $N$ th record selection out of 1,000 from each specialty group.

We attempted to optimize the response rate by (a) emphasizing the knowledge generation goal of the study and the affiliation with training programs at a large public medical school and offering to share results with respondents; (b) minimizing response burden with a quick and efficient survey (approximately $5 \mathrm{~min}$ in duration); and (c) offering a small incentive (online Amazon gift certificate) to complete the survey.

The total number of participants was 495 , but complete data were received from 371 respondents and thus all data reported hereinafter are from the 371 complete responders. Table 1 displays the characteristics of the 371 complete responders. Anecdotal reports suggest that technology challenges may have reduced response rates and survey completion rates. For example, some email solicitations were 
Table 1 Descriptive characteristics for provider survey sample (total $n=371$ )
${ }^{\text {a }}$ Forty-four states out of 50 represented

\begin{tabular}{|c|c|c|c|}
\hline Variable & & Number & Percentage \\
\hline \multirow[t]{2}{*}{ Gender } & Male & 194 & 52.3 \\
\hline & Female & 177 & 47.7 \\
\hline \multirow[t]{5}{*}{ Race/ethnicity } & Non-Hispanic White & 256 & 69 \\
\hline & Asian/Pacific Islander & 73 & 19.7 \\
\hline & Hispanic & 21 & 5.7 \\
\hline & African American & 13 & 3.5 \\
\hline & Mixed & 8 & 2.2 \\
\hline \multirow[t]{2}{*}{ Languages spoken } & Spanish & 59 & 15.9 \\
\hline & Other & 59 & 15.9 \\
\hline \multirow[t]{3}{*}{ Medical school location (top three locations) } & USA & 289 & 77.9 \\
\hline & Asia & 29 & 7.8 \\
\hline & India & 13 & 3.5 \\
\hline \multirow[t]{3}{*}{ Years in practice } & $<5$ & 56 & 15.1 \\
\hline & $5-20$ & 173 & 46.6 \\
\hline & $>20$ & 141 & 38 \\
\hline \multirow[t]{3}{*}{ State $^{\mathrm{a}}$ (top three) } & $\mathrm{CA}$ & 54 & 14.6 \\
\hline & NY & 32 & 8.6 \\
\hline & PA & 24 & 6.5 \\
\hline \multirow[t]{2}{*}{ Practice location } & Suburban & 152 & 41 \\
\hline & Urban, non inner city & 124 & 33.4 \\
\hline \multirow[t]{4}{*}{ Practice setting } & Hospital & 155 & 41.8 \\
\hline & Outpatient clinic & 308 & 83 \\
\hline & Affiliated with med school & 169 & 45.6 \\
\hline & Med school faculty & 130 & 35 \\
\hline \multirow[t]{4}{*}{ Primary specialty } & Family Medicine & 87 & 23.5 \\
\hline & Pediatrics & 108 & 29.1 \\
\hline & General Psychiatry & 84 & 22.6 \\
\hline & Child/Adol. Psychiatry & 92 & 24.8 \\
\hline
\end{tabular}

captured by spam filters and lack of wi-fi access would result in slow loading of the video, which may have resulted in partial completion.

\section{Procedures}

Participants were recruited in two waves of 4,000 each $(8,000$ total email invitations). Wave 1 physicians were sampled midweek and a follow-up reminder was sent 7 days later. Sampling procedures for wave 2 were exactly the same and began 1 month after wave 1 . The invitation included a brief general description of the study purpose as a study of provider practices (there was no mention of attention to race/ethnic disparities so as not to prime respondents regarding the specific research questions or hypothesis) and description of the general task (i.e., viewing one brief clinical vignette and answering questions about it). Clicking on the hyperlink to the survey took respondents to the Informed Consent page of the survey. Respondents were notified that advancing beyond the Informed Consent screen constituted an electronic signature to participate in the research project. Upon consenting, participants were asked basic demographic and patient characteristic questions, followed by viewing one of the randomly selected video clinical vignettes of approximately $2 \mathrm{~min}$ duration. The random assignment of video vignette conditions (i.e., race/ethnicity of the mother) was set to approximately $33 \%$ for each video. The final distribution was Hispanic $31 \%$, African American $33.2 \%$, and non-Hispanic White $35.8 \%$.

The survey questions assessing diagnostic and treatment decision-making followed the vignettes. Upon completion of the survey, respondents were offered the opportunity to register for an online Amazon.com gift certificate delivered to their email address of choice.

\section{Data Analysis}

Data analysis included basic descriptive data on participant characteristics and response frequencies to diagnostic and treatment recommendation survey questions. Primary 
hypotheses (effect of patient race/ethnicity on indicated diagnoses and treatment plans) were examined using Chi-square analyses. All analyses were conducted using SPSS 20. Significance tests were based on Pearson $\chi^{2}$ tests. Statistical significance was evaluated using .05-level two-sided designbased tests.

\section{Results}

Total Sample: Diagnostic and Treatment Decision-Making

\section{Diagnostic Assignment}

Table 2 lists all the frequencies for the total sample (far right column) and the frequencies within each of the groups by race/ethnicity of the mother in the clinical vignette. Across all respondents, the frequency of diagnostic assignment was as follows in order of frequency: ADHD $61.7 \%(n=229)$; oppositional defiant disorder (ODD) $48.2 \%(n=179)$; mood disorder (depression or bipolar) $37.5 \%(n=139)$; conduct disorder $21.0 \%(n=78)$. Eleven percent $(n=41)$ of the participants endorsed "no diagnosis," $28 \%(n=104)$ endorsed one diagnosis, and $60.9 \%(n=225)$ endorsed two or more diagnoses. As indicated in the "Research Methods" section, the vignette was designed to report symptoms meeting diagnostic criteria for ODD and sub-threshold symptoms of ADHD.

\section{Treatment Recommendations}

The vast majority of respondents $(98.3 \%, n=365)$ indicated that they would recommend psychosocial or psychotherapeutic treatment. The most commonly recommended types of psychosocial treatment were individual child treatment (82.7\%, $n=307)$, family therapy $(72.5 \%, n=269)$, and parent training $(60 \%, n=222)$. A minority of respondents recommended group treatment, $(13.2 \%, n=49)$ and mental health treatment for the parent only $(5.9 \%, n=22)$ as the specific treatment choice. These recommendations were not mutually exclusive and the majority of respondents selected more than one psychosocial treatment option.

A smaller majority of respondents $(60.3 \%, n=223)$ indicated that they would recommend pharmacologic treatment. When queried to specify what type of medication, $46.1 \%(n=$ $171)$ endorsed stimulants, $17.5 \%(n=65)$ antidepressants, $8.9 \%(n=33)$ mood stabilizers, and $4.9 \%(n=18)$ other medications which included atypical antipsychotics $(n=5)$ and "non-stimulant ADHD medication" $(n=5)$. These choices were not mutually exclusive; $44.1 \%(n=162)$ of respondents endorsed one medication, and $16.3 \%$ $(n=60)$ endorsed two or more.

Among the respondents who indicated that pharmacologic treatment was not indicated $(n=148)$, when asked for a reason, the majority $(86.5 \% ; n=128$ of 148$)$ endorsed that they would "Want to try other interventions first." Other responses endorsed by multiple respondents were "Medication is not indicated for this diagnosis" $(10.1 \%, n=15)$ and "Risks of medication outweigh potential benefit" $(8.8 \%, n=13)$.

Finally, $7 \%(n=26)$ of respondents recommended a school intervention and $3.5 \%(n=13)$ recommended a holistic health intervention. (These numbers were too small to run $X^{2}$ analyses due to small cell size).

Testing for an Effect of Patient Race on Diagnosis and Treatment Planning

\section{Diagnostic Assignment}

Chi-square tests were run to test for a group difference on rate of endorsement for any of the diagnostic options (including: ADHD, anxiety, conduct disorder, disruptive disorder NOS, mood disorders (bipolar and major depression), oppositional defiant disorder, none). There were no significant findings on any of these tests (all $p$ values $>.05$ ). To further explore any potential effects, the disruptive behavior diagnoses of conduct disorder, ODD, and disruptive disorder NOS were combined into a disruptive behavior disorder category. The Chi-square test on this distribution did not approach significance (Chisquare $=.68, d f=2, p=.711$ ).

\section{Treatment Recommendations}

The very high rate of recommendation for psychosocial treatment $(98 \%)$ overall was consistent across the race/ethnic groups; thus, there was no significant group difference (Chisquare $=2.27, d f=2, p=.32$ ). Virtually all physicians recommended psychosocial treatment regardless of which clinical vignette they viewed. There was greater variance in specific types of psychosocial treatment (e.g., individual child, family, parent training) as noted in Table 2, but there was no significant group difference in rates of recommendation for these types of psychosocial treatment (all $p$ values $>.30$ ).

There was variability on recommendation of psychopharmacological treatment with an overall rate of endorsement of $60 \%$. As noted in Table 2, among respondents who viewed the Hispanic vignette, $55.2 \%(n=64)$ recommended medication, among those viewing the African American vignette, $63.9 \%(n=78)$ recommended medication, and among those who viewed the non-Hispanic White vignette, $61.4 \%(n=81)$ recommended medication. These rates of endorsement did not represent statistically significant difference by group (Chisquare $=2.01, d f=2, p=.37$ ). There was one significant group difference on recommendation for anti-depressant medication. As noted in the summary table, anti-depressants were recommended more frequently for the non-Hispanic White patient (39.5\%; $n=32)$, compared to the African American ( $25.6 \%$, 
Table 2 Diagnostic assignment and treatment recommendation by race/ethnicity

\begin{tabular}{|c|c|c|c|c|c|c|c|c|}
\hline \multirow[t]{3}{*}{ Diagnostic and treatment selections } & \multicolumn{6}{|c|}{ Race/ethnicity of mother in vignette } & \multirow{2}{*}{\multicolumn{2}{|c|}{$\begin{array}{l}\text { Total } \\
N=371\end{array}$}} \\
\hline & \multicolumn{2}{|c|}{$\begin{array}{l}\text { Hispanic } \\
N=115\end{array}$} & \multicolumn{2}{|c|}{$\begin{array}{l}\text { African American } \\
N=123\end{array}$} & \multicolumn{2}{|c|}{$\begin{array}{l}\text { Non-Hispanic White } \\
N=133\end{array}$} & & \\
\hline & $N$ & $\%$ & $n$ & $\%$ & $n$ & $\%$ & $n$ & $\%$ \\
\hline \multicolumn{9}{|l|}{ Diagnostic Assignment } \\
\hline Oppositional defiant disorder & 50 & 43.5 & 62 & 50.4 & 67 & 50.4 & 179 & 48.2 \\
\hline Attention deficit hyperactivity disorder & 54 & 46.9 & 83 & 67.5 & 82 & 61.7 & 229 & 61.7 \\
\hline Mood disorder (depression, bipolar) & 34 & 29.6 & 51 & 41.4 & 54 & 40.6 & 139 & 37.5 \\
\hline Conduct disorder & 23 & 20.0 & 27 & 22.0 & 28 & 21.0 & 78 & 21.0 \\
\hline Anxiety disorder & 8 & 7.0 & 7 & 5.7 & 8 & 6.0 & 23 & 6.2 \\
\hline Autism disorder & 6 & 5.2 & 2 & 1.6 & 2 & 1.5 & 10 & 2.7 \\
\hline Disruptive behavior disorder (includes ODD, conduct, DBD NOS) & 69 & 60.0 & 79 & 64.2 & 86 & 64.7 & 234 & 63.1 \\
\hline No diagnosis & 17 & 14.7 & 9 & 7.3 & 16 & 12.0 & 42 & 11.3 \\
\hline \multicolumn{9}{|l|}{ Treatment recommendations } \\
\hline Any psychosocial therapy & 112 & 98.2 & 120 & 98.4 & 133 & 100 & 365 & 98.3 \\
\hline Individual child & 96 & 83.5 & 98 & 79.7 & 113 & 85.0 & 307 & 82.7 \\
\hline Family therapy & 85 & 73.9 & 87 & 70.7 & 97 & 72.9 & 269 & 72.5 \\
\hline Parent training & 75 & 65.2 & 69 & 56.1 & 78 & 59.0 & 222 & 60.0 \\
\hline Any pharmacologic treatment & 64 & 55.2 & 78 & 63.9 & 81 & 61.4 & 223 & 60.3 \\
\hline Stimulants & 49 & 42.6 & 63 & 51.2 & 59 & 48.0 & 171 & 46.1 \\
\hline Anti-depressants $^{\mathrm{a}}$ & 13 & 11.3 & 20 & 16.2 & 32 & 24.1 & 65 & 17.5 \\
\hline Mood stabilizers & 7 & 6.0 & 15 & 12.2 & 11 & 8.3 & 33 & 8.9 \\
\hline
\end{tabular}

${ }^{\mathrm{a}} X^{2}=6.86,(2) ; p=.03$

$n=20)$ and the Hispanic $(20.6 \%, n=13)$ patients (Chisquare $=6.86, d f=2, p=.03$ ). There were no significant group differences in frequency of recommendation of the stimulants or mood stabilizer medications ( $p$ values $>.4$ ).

\section{Exploratory Follow-up Analyses}

We conducted follow-up analyses to explore for physician characteristics that may have been associated with treatment recommendations. There were significant differences in treatment recommendations based on physician specialty. Specifically, psychiatrists (general and child/adolescent) were more likely to recommend pharmacological treatment compared to the Family Medicine and Pediatric physicians. Specifically, $72.5 \%(n=66)$ of child and adolescent psychiatrists, $67.5 \%(n=56)$ of general psychiatrists, $54.0 \%(n=47)$ of Family Medicine physicians, and $50.5 \%(n=54)$ of pediatricians recommended psychoactive medications. Exploratory three-way analyses to text for any effects of patient race/ethnicity by physician specialty for recommendation of psychopharmacology treatment did not reveal any significant effects. In addition, male physicians were significantly more likely to recommend medication compared to female physicians, but the specialty differences held within gender groups.

\section{Discussion}

This study was designed to investigate whether and how patient race/ethnicity may affect physician diagnostic and treatment decision-making for children with disruptive behavior problems when other factors are controlled. Results of this national web-based survey of physicians likely to care for these children (pediatricians, family physicians, general and pediatric psychiatrists) did not reflect evidence of major differences in diagnostic or treatment decision-making based on race/ethnicity of the patient's mother. Given the well-documented race/ethnic disparities in mental health service use, the authors expected to find a corresponding effect on physician decision-making for treatment. However, as multilevel models of health disparities emphasize, health service use is affected by many interacting factors. Race/ethnic disparities in mental health service use are likely attributed primarily to factors such as socioeconomic factors [8], family help-seeking attitudes and beliefs [37, 38], and funding or policy level factors [39]. Emerging support for the significant impact of funding level factors comes from studies demonstrating that race/ethnic disparities in unmet need decreased when insurance coverage is expanded in programs such as SCHIP (given that race/ethnic minority children are over-represented in the populations affected by these expansions) [40]. 
The mostly null findings in this study could also be interpreted as promising evidence that physicians' decisionmaking is not significantly influenced by race/ethnicity when other clinical and family context variables are held constant (such as socioeconomic status, parental employment, family composition, treatment motivation, etc.). For example, in another study examining the role of adult patient race/ ethnicity on decisions to prescribe medication, investigators found that patient characteristics such as occupation and perceived reliability with follow-up were more significantly associated with physician decision-making than the patient's race/ethnicity [27].

Almost all the research on race/ethnic disparities in children's mental health services has examined actual service use. Studies which test for race/ethnic differences in referral to services (as opposed to use) have, like the present study, demonstrated somewhat unexpected results. Some studies have found race/ethnic differences in mental health referral patterns for at-risk youth [41]. However, like the current study, one recent large national study examining the role of referral pathways in driving disparities in youth mental health service use also resulted in unexpected null findings across most analyses [11]. Specifically, the authors expected to find a significant racial/ethnic difference in identification of mental health problems and encouragement to seek services, but they found virtually no significant differences. The study included data on a nationally representative sample of 6,112 adolescents from across the USA and thus had more than adequate power to detect significant effects. The authors attributed the null findings to potential regional differences that may be obscured in the national sample (and may have driven the results of previous regional studies) and to changes over time in professionals' recognition of mental health problems across race/ethnic groups. They speculated that professional education efforts over recent decades may be decreasing biased or differential interpretation and recognition of behavioral health problems based on race/ethnicity, which is also a potentially reassuring hypothesis [11].

\section{Physician Decision-Making for Children with Disruptive Behavior Problems}

Despite the generally null findings related to the influence of race/ethnicity on physician decision-making, the descriptive findings are important. The range of responses to diagnostic assignment and treatment recommendations fell generally within predicted patterns for the clinical vignette stimuli. However, only approximately two thirds of the respondents assigned a disruptive behavior diagnosis to the child and only $48.2 \%$ assigned the specific disruptive disorder (ODD) that the vignette was designed explicitly to meet. The majority $(62.5 \%)$ of respondents assigned the diagnosis of attention deficit hyperactivity disorder, although the vignette did not include enough symptoms to meet diagnostic criteria. Many respondents assigned diagnoses of depression $(34.5 \%)$ or bipolar disorder $(7 \%)$. Although the vignette did not directly reflect symptoms of these mood disorders, irritability is a valid symptom criterion for depression, so this is not entirely surprising. This variability in diagnostic assignment is consistent with other studies documenting a lack of reliability and consistency in mental health diagnoses for children across reporters and/or diagnostic assessment methods [33, 42, 43].

Respondents' treatment recommendations were generally consistent with recommended best practices. Specifically, the majority of respondents recommended individual child psychotherapy, family therapy, and/or parent training therapy, and there are evidence-based treatment models for this patient population reflecting each of these approaches $[5,30]$. The psychopharmacological recommendations were also generally consistent with evidence-based approaches, with stimulants being the most common. It is notable that over $15 \%$ of physicians recommended two or more psychoactive medications for this patient (a Third grade student), reflecting some concerns that have been raised regarding increased rates of polypharmacy use in young children. Although studies of medication use in children with behavior problems identify rapidly rising rates of use of atypical antipsychotic medication (e.g., risperidone), very few physicians $(<10)$ indicated they would prescribe this medication.

The exploratory findings in this study, demonstrating that physicians trained in psychiatry were more likely to recommend medication compared to primary care physicians, are also not surprising given greater training and experience for the psychiatry specialists. The observed gender differences are also interesting, but somewhat confounded by specialty differences given an imbalance in the primary care fields versus psychiatry.

Finally, there was one lone statistically significant race/ ethnic effect on recommendation for antidepressant medication and the results were consistent with the study hypothesis. Frequency rates reveal that the non-Hispanic White patient was twice as likely as the Hispanic patient to receive a recommendation for antidepressants ( 24 vs. $11 \%$ ). A study examining prescription patterns for adults with depression similarly found higher rates of anti-depressant medication for NHW's but the comparison group was African Americans as opposed to Hispanics [21]. The finding is also indirectly consistent with results of other studies that have demonstrated that Non-Hispanic Whites are more likely to be diagnosed with mood disorders [17].

\section{Strengths and Limitations}

Strengths of this study include the collaborative researchpractice partnership team that worked together to develop the study aims and methods. The clinical vignette stimuli that 
was developed served the study design well in most regards in that the key outcomes for diagnostic decision-making and psychopharmacological treatment yielded sufficient variability to test for group differences (e.g., $60 \%$ of participants endorsed "yes" for medication). The standardization across race/ethnic groups was evaluated to be successful with equivalent ratings on constructs such as "attractiveness" and "believability." Ironically, it is possible that the lack of race/ethnic effects in this study may also reflect the care given to making sure the vignettes were equivalent in every respect except for the race/ethnicity of the mother. In other words, constructs that could be indirectly associated with race/ethnicity of the family (or potentially presumed by respondents) were explicitly controlled, such as health insurance coverage, treatment motivation, prior treatment history, and parental education level. However, given that the goal was to test an isolated effect of patient race/ethnicity, this equivalence was essential to the study design.

Of course, as noted in the introduction, one of the limitations of any study utilizing mock patient stimuli is that responses may not generalize to actual physician practice. We attempted to mitigate this limitation by working with a group of practicing physicians to refine the clinical vignette for maximum "ecological validity," (i.e., consistency with the "real world" of practice in terms of presenting problems, family context, etc.). Professional actresses were employed to deliver the vignette and their "believability" was rated very high. This study is the only one we are aware of that actually examines the potential role of physician decision-making in race/ethnic disparities of service use in children's mental health care. Most studies examine service use patterns (either through selfreport or record review) and thus cannot parcel out the role of physician treatment recommendations. Even studies of medication use have relied on prescriptions filled to identify disparities [21]; rates of prescriptions filled do not necessarily reflect provider practice.

One of the major significant limitations of the study is the disappointingly low response rate. This is often a challenge in broad survey research lacking significant funding for respondent compensation. As noted earlier, one of the challenges of this web-based survey format with embedded video stimuli was that the speed of accessing the video and the survey options varied depending on internet access and device used. Some potential participants did not complete the survey due to delays in accessing the video.

The final sample of 371 physician participants with complete data was diverse in terms of gender, age, race, etc. Physicians from 44 states and many different types of practices are included and the distribution across the targeted specialties most likely to treat these types of patients was relatively equivalent.

\section{Conclusion}

This study did not provide compelling evidence of an isolated effect of patient race/ethnicity on physician diagnostic or treatment decision-making for children with disruptive behavior problems and their families. There was only one significant finding demonstrating an effect of patient race/ethnicity on recommendation for antidepressant medication, with the nonHispanic White patient being twice as likely to receive this recommendation compared to the Hispanic patient. Given the well-established race/ethnic disparities in psychoactive medication use, more research is needed to identify factors at all levels of analysis that may be driving these disparities.

\section{Addendum: Vignette Script}

David's 3rd grade teacher told me to bring him to your clinic for an evaluation. He's been getting into a lot of serious trouble at school - and at home too. He seems angry all the time and he's been really aggressive. He was suspended from school last week for throwing a chair at the teacher-I couldn't believe he did that. I keep getting calls at work because he's been sent to the Principal's office at least five times in the past couple months - all for some kind of acting out. They tell me he often argues with the teacher and refuses to obey her-I believe it, because he's been like that at home too. He's got a quick temper and he gets too rough with his 2-year-old brother. I'm really worried that he's going to hurt him. He teases him all the time and blames him for all his own mistakes, like when he breaks something. It's really difficult to get him to help out at home- - He talks back and I have to keep reminding him over and over again. He's very forgetful and distracted. If I try to correct him for something, he gets really "touchy"-He over-reacts to anything he thinks is criticism.

We live with my parents now since my husband and I split up a few years ago. My mom has been really concerned about David too and she went to a parenting class with me a few months ago. We tried some of the strategies, but it hasn't really helped.

When I look back, it's clear that David's been a slightly challenging kid since day 1 , but he is smart and his grades were OK until recently. Of course, since kindergarten, his teachers have mentioned that he gets distracted easily, and he got inpatient, but nothing like he is now. This year his grades have gone way down and he often doesn't even do his work. I'm really worried because the teacher said he may not be able to stay at his school. You know, he's always been a healthy, active kid, but now he's stopped playing baseball and he really doesn't do anything with friends.

I'm at my wit's end with this kid. I am willing to try anything and my health insurance will cover whatever treatment you suggest. Thank you. 
Acknowledgments This study was funded by the Division of Child Development and Community Health within the Department of Pediatrics at the University of California, San Diego. (Grants: \#UCSD CDCH 20111952004 \& \#UCSD CDCH 20111952 006: Garland (PI)). Dr. Haine-Schlagel is an investigator with the Implementation Research Institute (IRI), at the George Warren Brown School of Social Work, Washington University in St. Louis, through an award from the National Institute of Mental Health (R25 MH080916) and Quality Enhancement Research Initiative (QUERI), Department of Veterans Affairs Contract, Veterans Health Administration, Office of Research \& Development, Health Services Research \& Development Service.

Conflict of Interest Statement Author Garland, Author Taylor, Author Brookman-Frazee, Author Baker-Ericzen, Author Haine-Schlagel, Author Liu, and Author Wong declare that they have no conflict of interest.

Informed Consent All procedures followed were in accordance with the ethical standards of the responsible committee on human experimentation (institutional and national) and with the Helsinki Declaration of 1975 , as revised in 2000(5). Informed consent was obtained from participants for being included in the study.

\section{References}

1. Institute of Medicine. Unequal treatment: confronting racial and ethnic disparities in health care (full printed version). Washington, DC: National Academies; 2003.

2. Kessler RC, Foster CL, Saunders WB, Stang PE. Social consequences of psychiatric disorders, I: educational attainment. Am J Psychiatry. 1995;152:10026-1032.

3. Garland AF, Hough R, McCabe K, Yeh M, Wood P, Aarons G. Prevalence of psychiatric disorders for youths in public sectors of care. J Am Acad Child Adolesc Psychiatry. 2001;40:409-18.

4. Copeland WE, Miller-Johnson S, Keeler G, et al. Childhood psychiatric disorders and young adult crime: a prospective, populationbased study. Am J Psychiatry. 2007;164:1668-75.

5. Eyberg SM, Nelson MM, Boggs SR. Evidence-based psychosocial treatments for children and adolescents with disruptive behavior. J Clin Child Adolesc Psychol. 2008;37:215-37.

6. Alegria M, Vallas M, Pumariega JJ. Racial and ethnic disparities in pediatric mental health. Child Adolesc Psychiatr Clin N Am. 2010;19:759-74.

7. Garland AF, Lau AS, Yeh M, McCabe KM, Hough RL, Landsverk JA. Racial and ethnic differences in utilization of mental health services among high-risk youths. Am J Psychiatry. 2005;162:1336-43.

8. Kataoka SH, Zhang L, Wells KB. Unmet need for mental health care among US children: variation by ethnicity and insurance status. Am J Psychiatry. 2002;159:1548-55.

9. Zito JM, Safer DJ, dos Reis S, Riddle MA. Racial disparity in psychotropic medications prescribed for youths with Medicaid insurance in Maryland. J Am Acad Child Adolesc Psychiatry. 1998;37:179-84.

10. Olfson M, Marcus SC, Weissman MM, Jensen PS. National trends in the use of psychotropic medications by children. J Am Acad Child Adolesc Psychiatry. 2002;41:514-21.

11. Alegria M, Lin JY, Green JG, Sampson NA, Gruber MJ, Kessler RC. Role of referrals in mental health service disparities for racial and ethnic minority youth. J Am Acad Child Adolesc Psychiatry. 2012;51:703-11.

12. Garland AF, Hough R, Landsverk J, McCabe K, Yeh M, Ganger W, et al. Racial/ethnic differences in use of mental health services for children in foster care. Child Serv Pol Res Pract. 2000;3:133-46.
13. Raphael JL, Beal AC. A review of the evidence for disparities in child vs. adult health care: a disparity in disparities. J Natl Med Assoc. 2010;102:684-91.

14. McCabe KM, Yeh M, Lau A, Garland AF, Hough RL. Racial/ethnic differences in caregiver strain and perceived social support among parents of youth with emotional and behavioral problems. Ment Health Serv Res. 2003;5:137-47.

15. Yeh M, McCabe K, Hough RL, Lau AS, Fakhry F, Garland AF. Why bother with beliefs?: examining relationships between race/ethnicity, parental beliefs about causes of child problems, and mental health service use. J Consult Clin Psychol. 2005;73:800-7.

16. Garland AF, Brookman-Frazee L, Gray E. The role of parent characteristics in community-based medication treatment for children with disruptive behavior problems. Community Ment Health J. 2013;49:507-14.

17. Del Bello MP, Lopez-Larson MP, Soutullo CA, Strakowski SM. Effects of race on psychiatric diagnosis of hospitalized adolescents: a retrospective chart review. J Child Adolesc Psychopharmacol. 2001;11:95-103.

18. Fabrega H, Ulrich R, Mezzich JE. Do Caucasian and Black adolescents differ at psychiatric intake? J Am Acad Child Adolesc Psychiatry. 1993;32:407-13.

19. Kilgus MD, Pumariega AJ, Cuffe SP. Influence of race on diagnosis in adolescent psychiatric inpatients. J Am Acad Child Adolesc Psychiatry. 1995;34:67-72.

20. Strakowski SM, Keck Jr PE, Arnold LM, Collins J, Wilson RM, Fleck DE, et al. Ethnicity and diagnosis in patients with affective disorders. J Clin Psychiatry. 2003;64:747-54.

21. Kuno E, Rothbard AB. Racial disparities in antipsychotic prescription patterns for individuals with schizophrenia. Am J Psychiatr. 2002;159:567.

22. Hudson J, Miller GE, Kirby JB. Explaining racial and ethnic differences in children's use of stimulant medications. Med Care. 2007;45: 1068-75.

23. Yeh M, McCabe K, Hough R, Dupuis D, Hazen A. Racial/ethnic differences in parental endorsement of barriers to mental health services for youth. Ment Health Serv Res. 2003;5(2):65-77.

24. van Ryn M, Fu SS. Paved with good intentions: do public health and human service providers contribute to racial/ethnic disparities in health? Am J Public Health. 2003;93:248-55.

25. van Ryn M, Hannan E, Burke J, Besculides M. An examination of factors associated with physician recommendation for revascularization. Washington, DC: American Public Health Association; 1999.

26. van Ryn M. Research on provider contribution to race/ethnicity disparities in medical care. Med Care. 2002;40:140-51.

27. Tamayo-Sarver JH, Dawson NV, Hinze SW, Cydulka RK, Wigton RS, Albert JM, et al. The effect of race/ethnicity and desirable social characteristics on physicians' decisions to prescribe opiod analgesics. Acad Emerg Med. 2003;10:1239-48.

28. Epstein SA, Hooper LM, Weinfurt KP, DePuy V, Cooper LA, Harless WG, et al. Primary care physicians' evaluation and treatment of depression: results of an experimental study using video vignettes. Med Care Res Rev. 2008;65:674-95.

29. Burk JP, Sher KJ. Labeling the child of an alcoholic: negative stereotyping by mental health professionals and peers. J Stud Alcohol. 1990;51:156-63.

30. Garland AF, Hawley KM, Brookman-Frazee LI, Hurlburt M. Identifying common elements of evidence-based psychosocial treatments for children's disruptive behavior problems. J Am Acad Child Adolesc Psychiatry. 2008;47:505-14.

31. Comer JS, Olfson M, Mojtabai R. National trends in child and adolescent psychotropic polypharmacy in office-based practice, 1996-2007. J Am Acad Child Adolesc Psychiatry. 2010;49: $1001-10$. 
32. AACAP Work Group on Quality Issues. Practice parameter on the use of psychotropic medication in children and adolescents. J Am Acad Child Adolesc Psychiatry. 2009;48:961-73.

33. Jensen AL, Weisz JR. Assessing match and mismatch between practitioner-generated and standardized interview-generated diagnoses for clinic-referred children and adolescents. J Consult Clin Psychol. 2002;70:158-68.

34. Swanson JM, Kraemer HC, Hinshaw SP, et al. Clinical relevance of the primary findings of the MTA: success rates based on severity of ADHD and ODD symptoms at the end of treatment. J Am Acad Child Adolesc Psychiatry. 2002;40:168-79.

35. Zito J, Safer D, dos Reis S, Magder L, Gardner J, Zarin D. Psychotherapeutic medication patterns for youths with attention-deficit/hyperactivity disorder. Arch Pediatr Adolesc Med. 1999;153: 1257-63.

36. Duffy F, Narrow W, Rae D, et al. Concomitant pharmacotherapy among youths treated in routine psychiatric practice. J Child Adolesc Psychopharmacol. 2005;15:12-25.

37. Angold A, Erkanli A, Farmer EMZ, Fairbank JA, Burns BJ, Keeler $\mathrm{G}$, et al. Psychiatric disorder, impairment, and service use in rural
African American and White youth. Arch Gen Psychiatry. 2002;59: 893-901.

38. Shin S, Brown T. Racial and ethnic disparities in caregiver strain and the use of child mental health services: a structural equation model. Psychiatr Serv. 2005;2009(60):1039-45.

39. Croft B, Parish SL. Care integration in the patient protection and affordable care act: implications for behavioral health. Adm Policy Ment Health Ment Health Serv Res. 2012;35:21-37.

40. Dick AW, Brach C, Allison RA, et al. SCHIP's impact in three states: how do the most vulnerable children fare? Health Aff (Millwood). 2004;23:63-75.

41. Langrehr KJ. Racial distinctions in the psychosocial histories of incarcerated youth. Psychol Serv. 2011;8:23-35.

42. Galanter CA, Patel VL. Medical decision making: a selective review for child psychiatrists and psychologists. J Am Acad Child Adolesc Psychiatry. 2005;46:675-89.

43. Lewczyk CM, Garland AF, Hurlburt M, Gearity J, Hough RL. Comparing DISC-IV and clinician diagnoses among youths receiving public mental health services. J Am Acad Child Adolesc Psychiatry. 2003;42:349-56. 